\title{
14. Mutagen Formation after Heating of Mixtures of Guanidino Compounds, Amino Acids and Glucose
}

\author{
By Atsuko OGURI, ${ }^{*)}$ Minako NAGAO, ${ }^{* *)}$ Nobuhiko ArAKAWA,***) \\ Takashi SugImuRA, M. J. A., ${ }^{*)}$ and Keiji WAKABAYASHI*)
}

(Communicated May 12, 1994)

\begin{abstract}
The effect of guanidino compounds other than creatin(in)e on mutagen formation was tested after heating with amino acid and glucose in a solution at $128^{\circ} \mathrm{C}$ for $2 \mathrm{~h}$. The mutagenicity of a heated mixture of creatine, glycine and glucose to Salmonella typhimurium TA98 with S9 mix was 630 per $\mathrm{mg}$ of heated material. When L-arginine was mixed with glycine plus glucose, 39 revertants of TA98 were induced per mg of heated material. Other guanidino compounds such as $N^{\mathrm{G}}$-methyl-L-arginine, 1-methylguanidine, aminoguanidine and guanidine also yielded mutagens, inducing 6.0 64 revertants per $\mathrm{mg}$ of heated material. When guanidino compounds were heated with L-phenylalanine and glucose, mixtures also yielded mutagenicity. The mutagenicity of a heated mixture of creatine, L-phenylalanine and glucose to TA98 with S9 mix was 160 per $\mathrm{mg}$ of heated material. L-Arginine, $N^{\mathrm{G}}$-methyl-L-arginine, aminoguanidine and guanidine gave 1.9 21 revertants per $\mathrm{mg}$ of heated material. With the addition of 1-methylguanidine, a very potent mutagenicity of 200 per $\mathrm{mg}$ of heated material was observed.
\end{abstract}

Key words: Guanidino compounds; creatine; L-arginine; mutagen.

Various kinds of mutagenic and carcinogenic heterocyclic amines (HCAs) are isolated from cooked meat and fish. ${ }^{1,2)}$ It has been reported that precursors of 2-amino-3methylimidazo[4,5-f]quinoline (IQ)-type HCAs, having 2-aminoimidazole moiety as a common structure, are creatin(in)e, amino acids and sugars in meat and fish. Actually, 2-amino-3,8-dimethylimidazo[4,5-f]quinoxaline (MeIQx) was formed by heating a mixture of creatinine, glycine and glucose, and of creatinine, L-threonine and glucose. ${ }^{3), 4)}$ 2-Amino-1-methyl-6-phenylimidazo[4,5- $b]$ pyridine (PhIP) was found in heated mixtures of creatin(in)e, L-phenylalanine and glucose. ${ }^{5), 6)}$ Thus, it is suggested that an aminoimidazole moiety of IQ-type HCAs is derived from creatin(in)e, and remaining parts are from Maillard reaction products of amino acids and sugars.

IQ-type HCAs are reported to be present not only in cooked meat and fish but also heated materials containing no meat products. For example, IQ was detected in cigarette smoke condensate ${ }^{7)}$ and MeIQ in coffee. ${ }^{8)}$ Since creatine and creatinine are not present in tobacco leaves and coffee beans, components other than creatin(in)e, must be involved in the formation of IQ-type HCAs in cigarette smoke and coffee. It is most likely that guanidino compouds other than creatin(in)e including L-arginine and $N^{\mathrm{G}}$-methyl-Larginine, may serve as a precursor to provide aminoimidazole moiety during heating process. The effect of five guanidino compounds other than creatin(in)e on mutagen

*) Biochemistry Division, National Cancer Center Research Institute, 5-1-1 Tsukiji, Chuo-ku, Tokyo 104, Japan.

**) Carcinogenesis Division, National Cancer Center Research Institite, 5-1-1 Tsukiji, Chuo-ku, Tokyo 104, Japan.

***) Department of Food and Nutrition, Ochanomizu University, 2-1-1 Otsuka, Bunkyo-ku Tokyo 112, Japan. 
formation in heated material of guanidino compound, amino acid and glucose was examined and compared with that of creatine.

A mixture of $1 \mathrm{mmol}$ of glycine and $0.5 \mathrm{mmol}$ of glucose with or without $1 \mathrm{mmol}$ guanidino compound in $3 \mathrm{ml}$ of diethylene glycol containing $14 \%$ water was heated at $128^{\circ} \mathrm{C}$ for $2 \mathrm{~h}$, as described previously. ${ }^{3)}$ The heated mixture was diluted with $180 \mathrm{ml}$ of water and treated with separate $0.5 \mathrm{~g}$ portions of blue rayon three times (Funakoshi Pharmaceutical Co. Ltd., Tokyo). After washing the blue rayon with water, the adsorbed materials to the rayon were eluted with $240 \mathrm{ml}$ of methanol-ammonia water $(50: 1$, v/v) two times, as reported previously. ${ }^{7)}$ This eluate was evaporated and the residue was dissolved in $2 \mathrm{ml}$ of dimethyl sulfoxide, and its mutagenicity was tested.

The pre-incubation methods, ${ }^{9)}$ a modification of the Ames mutagenicity testing method, was adopted to test the mutagenicity of the samples to Salmonella typhimurium TA98 with or without S9 mix. The S9 mix contained $30 \mu \mathrm{l}$ of S9, prepared from the livers of rats treated with polychlorinated biphenyls. Mutagenic activity was calculated from the linear portion of the dose-response curve, which was obtained using three or four doses in duplicate plates.

Table I shows mutagenicity of heated mixtures to S. typhimurium TA98 in the presence of S9 mix. When creatine monohydrate (Wako Pure Chemical Industries, Osaka), glycine and glucose were mixed and heated at $128^{\circ} \mathrm{C}$ for $2 \mathrm{~h}, 630$ revertants of TA98 were induced per $\mathrm{mg}$ of heated material. This mutagenicity is almost the same as that in the previous report ${ }^{3)}$ of the heated mixture of creatinine, glycine and glucose, in which MeIQx was detected. By addition of L-arginine (Wako Pure Chemical Industries), instead of creatine, to the mixture of glycine plus glucose, 39 revertants were observed per $\mathrm{mg}$ of heated material. In the case of $N^{\mathrm{G}}$-methyl-L-arginine acetate salt (Sigma Chemical Co., St. Louis, MO), 29 revertants per $\mathrm{mg}$ of heated material were induced. The mutagenicities of heated mixtures with addition of 1-methylguanidine hydrochloride (Tokyo Chemical Industry Co., Tokyo) and aminoguanidine hydrochloride (Wako Pure Chemical Industries) were $160 \%$ and $85 \%$ that of the heated mixture of L-arginine, glycine and glucose, respectively. Among heated mixtures with guanidino compounds, a heated mixture with guanidine (Tokyo Chemical Industry Co.) showed the lowest mutagenicity, being 6.0 per $\mathrm{mg}$ of heated material. On the other hand, mutagenicity of a mixture of glycine and glucose heated without guanidino compounds, induced only 7.6 revertants per $\mathrm{mg}$ of heated material. In the absence of S9 mix, mutagenicity was not observed in any heated samples up to the dose of $10 \mathrm{mg}$ heated material.

Table I. Mutagenicities of mixtures of glycine and glucose heated with or without guanidino compounds

\begin{tabular}{lc}
\hline & $\begin{array}{c}\text { Mutagenicity } \\
\text { a) }\end{array}$ \\
& (Revertants/mg heated material) \\
\hline Creatine + glycine + glucose & 630 \\
L-Arginine + glycine + glucose & 39 \\
$N^{\mathrm{G}}$-Methyl-L-arginine + glycine + glucose & 29 \\
1-Methylguanidine + glycine + glucose & 64 \\
Aminoguanidine + glycine + glucose & 33 \\
Guanidine + glycine + glucose & 6.0 \\
Glycine + glucose & 7.6 \\
\hline
\end{tabular}

a) Mutagenicity was tested in S. typhimurium TA98 with S9 mix, and number of revertants is indicated as an average obtained from two independent experiments. 
Since PhIP has been reported to be formed by heating a mixture of creatin(in)e, L-phenylalanine and glucose, ${ }^{5), 6)}$ mutagen formation in heated mixtures of guanidino compounds, L-phenylalanine and glucose was tested. Table II shows mutagenicities of mixtures of L-phenylalanine and glucose heated with or without addition of guanidino compounds. By heating a mixture of creatine, L-phenylalanine plus glucose, 160 revertants of TA98 were induced per mg of heated material in the presence of S9 mix. When creatine was replaced by L-arginine and $N^{\mathrm{G}}$-methyl-L-arginine, the mutagenicities of their heated mixtures were 21 and 19 per $\mathrm{mg}$ of heated material, respectively. Interestingly, the mutagenicity by the addition of 1-methylguanidine was higher than that of creatine, inducing 200 revertants per $\mathrm{mg}$ of heated material. A heated mixture of aminoguanidine, L-phenylalanine plus glucose gave 13 revertants of TA98 per $\mathrm{mg}$ of heated material. The mutagenicity of a heated mixture of guanidine, L-phenylalanine and glucose was almost 10-fold less than that with L-arginine. A heated mixture of L-phenylalanine and glucose without guanidino compound induced only 8.2 revertants per $\mathrm{mg}$ of heated material, and this mutagenicity was lower than those of heated mixtures with guanidino compounds excluding guanidine. Moreover, no mutagenicity was observed in any samples when tested with $S$. typhimurium TA98 in the absence of metabolic activation system (S9 mix) up to the dose of $10 \mathrm{mg}$ heated material.

In the present study, we found that five guanidino compounds other than creatin(in)e served as precursors to produce mutagenic aromatic compounds, when those guanidino compounds were mixed with amino acids and glucose and then heated. L-Arginine and its analogue, $N^{\mathrm{G}}$-methyl-L-arginine, are widely distributed in animal and plant materials. ${ }^{10}$ ) The level of L-arginine is comparable to that of creatine in beef. In the case of $N^{\mathrm{G}}$-methyl-L-arginine, its content is less than one thousandth that of creatine. Methylguanidine may be produced from L-arginine and $N^{\mathrm{G}}$-methyl-L-arginine during heating process. Thus, the guanidino compounds, especially L-arginine, presumably provide aminoimidazole moiety and contribute to the formation of mutagens including heterocylic amines in cooked meat and fish as well as heated materials containing no meat products. Recently, Knize et al. proposed that L-arginine must be involved in mutagen formation in cooked grain foods. ${ }^{11)}$ It is very important to determine what type of mutagenic compounds are present in the heated mixtures of guanidino compounds other than creatine, amino acids and glucose used in the present experiments.

Acknowledgements. This study was supported by Grants-in-Aid for Cancer Research from the Ministry of Health and Welfare of Japan, and grants from the Bristol-Myers Squibb Foundation and the Smoking Research Foundation.

Table II. Mutagenicities of mixtures of L-phenylalanine and glucose heated with or without guanidino compounds

\begin{tabular}{lc}
\hline & $\begin{array}{c}\text { Mutagenicity }^{\text {a) }} \\
\text { (Revertants/mg heated material) }\end{array}$ \\
\hline Creatine + L-phenylalanine + glucose & 160 \\
L-Arginine + L-phenylalanine + glucose & 21 \\
$N^{\mathrm{G}}$-Methyl-L-arginine + L-phenylalanine + glucose & 19 \\
1-Methylguanidine + L-phenylalanine + glucose & 200 \\
Aminoguanidine + L-phenylalanine + glucose & 13 \\
Guanidine + L-phenylalanine + glucose & 1.9 \\
L-Phenylalanine + glucose & 8.2 \\
\hline
\end{tabular}

a) The average of two independent experiments in S. typhimurium TA98 with S9 mix. 


\section{References}

1) Sugimura, T. (1986): Science, 233, 312-318.

2) Wakabayashi, K. et al. (1992): Cancer Res. (suppl.), 52, 2092s-2098s.

3) Jägerstad, M. et al. (1984): Mutat. Res., 126, 239-244.

4) Jägerstad, M. et al. (1991): ibid., 259, 219-233.

5) Shioya, M. et al. (1987): ibid., 191, 133-138.

6) Skog, K., and Jägerstad, M. (1993): Carcinogenesis, 14, 2027-2031.

7) Yamashita, M. et al. (1986): Jpn. J. Cancer Res. (Gann), 77, 419-422.

8) Kikugawa, K., Kato, T., and Takahashi, S. (1989): J. Agric. Food Chem., 37, 881-886.

9) Yahagi, T. et al. (1977): Mutat. Res., 48, 121-130.

10) Paik, W. K., and Kim, S. (1975): Adv. Enzymol., 42, 227-286.

11) Knize, M. G. et al. (1994): Fd Chem. Toxic., 32, 55-60. 